\title{
Health Beliefs and Preventive Behaviors Among Adults During the Early COVID-19 Pandemic in the United States: a Latent Class Analysis
}

\author{
Emily Smail ${ }^{1}$ (D) Kristin E. Schneider ${ }^{1} \cdot$ Stephanie M. DeLong $^{2} \cdot$ Kalai Willis $^{3} \cdot$ Renata Arrington-Sanders $^{2,4,5}$. \\ Cui Yang ${ }^{5} \cdot$ Kamila A. Alexander $^{6} \cdot$ Renee M. Johnson $^{1,2}$
}

Accepted: 16 June 2021 / Published online: 17 July 2021

(c) Society for Prevention Research 2021

\begin{abstract}
The objectives of this study are to (1) characterize patterns of preventive behaviors 3 months after the COVID-19 pandemic was declared a national emergency in the USA and (2) identify how health beliefs (e.g., perceived risk of infection, perceived risk of death upon infection, and perceived effectiveness of CDC-recommended preventive behaviors) and sociodemographic characteristics are associated with preventive behaviors. Data were obtained from two waves of the Understanding America Study (UAS) conducted in March (wave 1) and May to June of 2020 (wave 2) (n=4445); UAS is a nationally representative panel of US adults. We conducted a latent class analysis (LCA) using wave 2 data to identify our outcome, patterns of 10 COVID-preventive behaviors (e.g., wearing a facemask, handwashing, social distancing), and then used a three-step regression (R3STEP) to test associations between the likelihood of class membership with (1) health beliefs and sociodemographic characteristics (age, sex, race/ethnicity, and educational attainment) in bivariate models and (2) health beliefs adjusted for sociodemographic characteristics in multivariate models. The LCA identified a three-class model of preventive behaviors characterized by high likelihood of engagement in the set of preventive behaviors ("high"), low likelihood of the preventive behaviors ("low"), or engagement in some behaviors ("mixed"). Respondents of older age (i.e., age 50 or older) and those with higher levels of educational attainment (i.e., a 4-year college degree or higher) were less likely to be in the low engagement versus the mixed engagement class compared to those who are younger (18-29) and have lower levels of educational attainment (i.e., high school), respectively. Women (compared to men) and respondents who were Black and/or Hispanic/ Latinx (compared to White) were more likely to be in the high (vs. mixed) engagement class. In separate models adjusted for sociodemographic characteristics, respondents with a high perceived risk of infection, high perceived risk of death, and high perceived effectiveness of COVID-preventive behaviors were statistically significantly less likely to be in the low engagement relative to the mixed engagement class. Engagement in COVID-preventive behaviors varies by sociodemographic characteristics (i.e., age, sex, race/ethnicity and educational attainment) and health beliefs (i.e., perceived risk of infection, perceived risk of death, and perceived effectiveness of CDC-recommended behaviors). Our findings highlight the potential utility of using health beliefs to inform targeted prevention efforts to help reduce the spread of COVID-19 and future pandemics.
\end{abstract}

Keywords Health belief model $\cdot$ Health beliefs $\cdot$ Health behavior $\cdot$ COVID-19 $\cdot$ Prevention

Emily Smail

esmail1@jhu.edu

1 Department of Mental Health, Bloomberg School of Public Health, Johns Hopkins University, Room 897, 624 N Broadway, Baltimore, MD 21205, USA

2 Department of Epidemiology, Bloomberg School of Public Health, Johns Hopkins University, 624 N Broadway, Baltimore, MD 21205, USA

3 Department of Population, Family and Reproductive Health, Bloomberg School of Public Health, Johns Hopkins University, 624 N Broadway, Baltimore, MD 21205, USA
4 Department of Pediatrics, Division of Adolescent and Young Adult Medicine, 200 N. Wolfe Street, Baltimore, MD 21287, USA

5 Department of Health, Behavior and Society, Bloomberg School of Public Health, Johns Hopkins University, $624 \mathrm{~N}$ Broadway, Baltimore, MD 21205, USA

6 School of Nursing, Johns Hopkins University, 525 N. Wolfe St, 417, Baltimore, MD 21205, USA 


\section{Introduction}

The emergence and transmission of SARS-CoV-2 in the USA has introduced unprecedented challenges that have drastically changed daily life. As of April 2021, over a year after the pandemic was declared a national emergency in March 2020 (Raifman et al., 2020), more than 32.1 million people in the US have been infected with SARS-CoV-2 and there have been more than 572,000 deaths from complications of COVID-19 (Johns Hopkins Coronavirus Resource Center, 2020). Several behaviors to prevent transmission have been recommended by the Centers for Disease Control and Prevention (CDC), including social distancing, handwashing, and wearing a facemask (CDC, 2020). To control the spread of COVID-19, prevention scientists need to develop public health campaigns to promote preventive behaviors. We examine the psychosocial determinants of COVID-preventive behaviors in this study; our findings can inform communication campaigns and other behavior change efforts to reduce the spread of SARS-CoV-2.

\section{Applying the Health Belief Model to COVID-19 Preventive Behaviors}

The Health Belief Model (HBM) is a useful framework for considering factors associated with likelihood of engaging in COVID-related preventive behaviors (Glanz et al., 2015). The framework posits that motivation for preventive behaviors is determined by health beliefs, including (1) "perceived susceptibility" to the health condition in the absence of preventive behavior; (2) "perceived severity," or one's opinion of how bad it would be to have the health condition; and (3) "perceived benefits," or positive outcomes resulting from compliance with the recommended behavior. The model suggests that the likelihood of preventive behavior increases with higher levels of perceived susceptibility, perceived severity, and perceived benefits, provided that the behavior is relatively easy and there are no major barriers to action, such as high financial cost (Glanz et al., 2015; Rosenstock, 1974). The large body of knowledge on health beliefs and health behavior suggest that HBM could be a useful lens for investigating COVID-preventive behaviors. HBM was developed in the 1950s to understand and promote free tuberculosis screenings and is a particularly useful framework for simple behaviors (Rosenstock, 1974), such as mask wearing and handwashing.

\section{Behavioral Recommendations for Preventing COVID-19}

States began to enact stay-at-home orders and school closures to slow the spread of SARS-CoV-2 in mid-March
2020, and public health organizations emphasized the need for social distancing and handwashing. Experts estimated that the spread of SARS-CoV-2 could be substantially reduced if all people in the USA wore masks in public spaces and washed their hands often. By April 3, 2020, $\mathrm{CDC}$ issued a recommendation for wearing masks when indoors and away from home (Raifman et al., 2020).

Several studies investigated COVID-19 preventive practices during the early months of the US outbreak. In April 2020, Goldberg et al. (2020) used data from several market research panels and concluded that there had been increases in mask wearing and mask buying among US adults following CDC's issuance of recommendations. In May 2020, a CDC web-based survey found that $80 \%$ of adults supported social distancing and disapproved of gatherings with 10 or more people. The CDC study (2020) showed that majority of participants reported following stay-at-home orders (77\%), social distancing recommendations $(80 \%)$, and guidance to avoid large groups $(86 \%)$.

Research indicates that compliance with COVID-prevention recommendations in the earlier stages of the pandemic is inconsistent across contexts, population groups, and geographical areas (Goldberg et al., 2020; Wise et al., 2020), and there are also differences in rules, norms, and enforcement across locales. Patterns of adoption may be associated with health beliefs, such as the perceived likelihood of being infected (Wise et al., 2020), with demographic characteristics, including sex, age, and race (Alsan et al., 2020), as well as with health literacy, news and media consumption, and political affiliation (Grossman et al., 2020). For example, Alsan et al. (2020) reported that-relative to older adultsyoung adults were less likely to know how SARS-CoV-2 was spread and less likely to adopt preventive behaviors, such as handwashing. In addition, engagement in prevention behaviors may be influenced by systemic issues that heighten vulnerability of demographic subgroups, particularly racial and ethnic minorities. For example, mask-wearing has been cited as a concern for both Black and Asian individuals, who fear they will be subject to racial profiling or stereotyping (Zine, 2020). However, less is known about patterns of adoption for socially influenced prevention behaviors, such as cancelling travel and social distancing, and whether certain populations differentially adopt behaviors based on convenience or ability. Emerging research highlights a need to enhance understanding of how health beliefs, sociodemographic characteristics, and other factors are associated with COVID-19 preventive behaviors.

\section{The Current Study}

There is a need to develop a more comprehensive understanding of COVID-preventive behaviors and health beliefs, and how behaviors and beliefs vary across population 
subgroups. In this study, we use the HBM to conceptualize the psychosocial determinants of COVID-related preventive behaviors among US adults from mid-May to mid-June of 2020. Our objectives are to (1) characterize patterns of preventive behaviors 3 months after the COVID-19 pandemic was declared a national emergency in the USA and (2) identify whether health beliefs (e.g., perceived risk of infection, perceived risk of death upon infection, and perceived effectiveness of CDC-recommended preventive behaviors) and sociodemographic characteristics are associated with these preventive behaviors.

\section{Methods}

\section{Study Sample}

Data for this study on health behaviors to prevent COVID19 come from the Understanding America Study (UAS). UAS participants were selected using address-based sampling, in which postal records are used to select a random sample from a listing of residential addresses (Lavrakas, 2008). Eligible individuals include adults (18 and older) in the contacted households. The UAS panel consists of ten nationally representative cohorts (the University of Southern California, 2020) enrolled between 2014 and 2020. The current analysis uses data from two waves of a longitudinal study addressing the COVID-19 pandemic. Data for the exposure variables come from the first wave, which was administered from March 10 through March 31, 2020 ("UAS 230"); data for the outcome variables come from the second wave, which was administered from May 27 through June 23, 2020 (“UAS 246”).

\section{Measurement of Study Variables}

The outcome variable was level of engagement in a set of behaviors to prevent COVID-19. Data were collected after states across the nation had lifted stay-at-home orders (Raifman et al., 2020), meaning survey respondents could engage in preventive behaviors voluntarily. The question stem for preventive behaviors was: "Which of the following have you done in the last seven days to keep yourself safe from the coronavirus?," and items included (1) washed your hands with soap or used hand sanitizer several times per day; (2) stockpiled food or water; (3) avoided contact with people who could be high-risk; (4) avoided public spaces, gatherings, or crowds; (5) prayed; (6) avoided eating at restaurants; (7) wore a mask or face covering; (8) worked or attended school from home; (9) canceled or postponed work or school activities, including work travel, and (10) canceled or postponed personal or social activities, including travel for pleasure. Response options were yes and no. We considered the latter three preventive behaviors to be social in nature because they involve mitigating risk among personal friends and colleagues and because they reflect restricting physical contact among one's social network, i.e., "social distancing." As described in the analysis section below, we used responses to derive a single, latent variable representing level of engagement in preventive behaviors.

The three exposure variables are beliefs about (1) one's risk of infection (i.e., perceived susceptibility), (2) one's risk of death upon infection (i.e., perceived severity), and (3) the effectiveness of the behaviors for preventing infection (i.e., perceived benefits). Data on these variables were collected in March 2020. The item on perceived susceptibility asked: "What is the chance that you will get the coronavirus in the next three months?"; the item assessing perceived severity asked: "If you do get the coronavirus, what is the percent chance you will die from it?" Respondents were asked to respond using a scale of $0-100 \%$. Forty-two percent reported perceived susceptibility above $20 \%$ and one-third indicated perceived severity above $20 \%$. Based on the distribution of responses, we categorized perceived susceptibility and severity as no risk (0\%), low risk (1-20\%), and moderatehigh risk (21-100\%).

To assess perceived benefits, participants were asked to rate three COVID-preventive behaviors on a 4-point scale, i.e., extremely ineffective (1), somewhat ineffective (2), somewhat effective (3), and extremely effective (4). The question stem asked, "How effective are the following actions for keeping you safe from coronavirus?"; specific items were "Wearing a face mask," "Washing your hands with soap and hand sanitizer frequently," and "Avoiding public spaces, gatherings, and crowds." A total score was calculated by summing responses to the three questions (range 3-12). Respondents also had the option to indicate they were unsure. Participants who reported that they were "unsure" about the effectiveness of three recommended behaviors were excluded from the analysis because it was unclear how they rate perceived benefits of the behaviors. Fourteen percent of the sample $(n=956)$ were excluded based on this criterion. Analyses were conducted to check whether findings were sensitive to exclusion of these respondents; there was no indication that restricting the sample changed the results.

All covariates were measured in March 2020. Covariates included several sociodemographic characteristics: age category (18-29, 30-39, 40-49, 50-59, and 60+), sex (female or male), educational attainment (high school degree or below, attended some college or received a 2-year degree, bachelor's degree, or graduate degree), race/ethnicity, and state of residence classified according to US Census regions (Northeast, Midwest, South, and West) (US Census Bureau, 2013). Race/ethnicity categories included non-Hispanic White, non-Hispanic Black, Hispanic/Latino of any race, and all other groups, which include those who were American 
Indian or Alaska Native, Asian, Native Hawaiian or Pacific Islander, or multi-racial.

\section{Statistical Analyses}

The analysis was restricted to 4445 participants with complete information on all analytic variables at wave 1 and latent class indicators at wave 2. Data structuring occurred in Stata 15 (Statacorp, 2017). Survey weights were applied to align sample distributions of key demographic characteristics (e.g., sex, race/ethnicity) to their population counterparts based on the Basic Monthly Current Population Survey (US Bureau of Labor Statistics, 2021).

First, we used latent class analysis (LCA) to characterize level of engagement in preventive behavior; we determined the number and structure of latent classes. Models of 2 to 6 classes were estimated, and we decided on the optimal number of latent classes based on model fit statistics (i.e., Akaike information criterion or AIC, Bayesian information criterion or BIC, and likelihood ratio test or LRT), class size, and substantive interpretability. To test for potentially different results based on the exclusion of behaviors with limited evidence of effectiveness, we ran sensitivity analyses excluding the behaviors "prayed" and "stockpiled food or water."

Next, we assessed the relationship between level of engagement in preventive behaviors (outcome) with health beliefs and sociodemographic characteristics. We used the R3STEP method to estimate the association membership in latent classes of preventive behavior with health beliefs and sociodemographic characteristics. To account for potential misclassification, the probability of membership in each class is estimated for each participant. Then, when assessing association between correlates and classes, class membership is weighted for uncertainty based on the probabilities of belonging to other classes. This method uses multinomial regression, and measures of effect are presented as odds ratios and 95\% confidence intervals (Asparouhov \& Muthén, 2014). Latent class modeling was performed in Mplus 8 (Muthen \& Muthen, 2017).

\section{Results}

\section{Sample Characteristics}

As shown in Table 1, one-half of the respondents were female and two-thirds were non-Hispanic White $(n=4445)$. Thirty-one percent were aged 60 or older, and one-third had an education attainment level of high school or less. About one-fifth of participants lived in the Midwest and Northeast, one-quarter lived in the West, and the remaining participants lived in the South. Seventeen percent evaluated their 3-month risk of contracting SARS-CoV-2 as $0 \%$, and $21 \%$ reported evaluated their risk of dying if they contracted the virus as $0 \%$. Forty-two percent of the sample reported low perceived risk (i.e., $<20 \%$ chance) for contracting the virus, and 55.6\% reported low perceived risk for dying upon infection.

\section{Classes of Preventive Behaviors}

There was a wide range of past 7-day engagement in behaviors to keep safe from SARS-CoV-2 in May-June 2020. Nearly all individuals reported frequent handwashing (93\%); the majority reported wearing a facemask (87.7\%), avoiding contact with people at high risk $(79.6 \%)$, avoiding public spaces and crowds $(76.2 \%)$, avoiding eating at restaurants (71.6\%), and praying $(60.2 \%)$; and less than onehalf reported canceling social activities (48.7\%), working from home (45.5\%), canceling social activities (26.4\%), and stockpiling food (12.1\%). The LCA revealed three classes of preventive behaviors: a high-engagement class (class 1), a mixed-engagement class (class 2), and a low-engagement class (class 3). Models with additional classes (i.e., 4-6) were unstable due to the sample size of the smallest class (Nylund-Gibson \& Choi, 2018). Fit statistics are presented in Table 2, and probabilities of each behavior by class are presented in Fig. 1. Around one-quarter (24.3\%) were in the high-engagement class and approximately one-fifth (21.8\%) were in the low-engagement class. Respectively, these two classes have the highest and lowest likelihood of engaging in all preventive behaviors. The majority of participants (53.9\%) were in the mixed-engagement class. Respondents in this class are distinguished from the high-engagement class based on their limited likelihood of engagement in preventive behaviors designated as social in nature, i.e., working from home, cancelling work or school activities, and canceling personal activities. Results were not sensitive to the exclusion of praying or stockpiling food, so we proceeded with the full model of prevention behaviors.

\section{Sociodemographic Characteristics and Classes of Preventive Behaviors}

The mixed engagement class was used as a comparison class in analyses to assess likelihood of class membership in association with health beliefs and sociodemographic characteristics; this class was selected based on its unique pattern of behaviors. Table 3 shows associations between sociodemographic characteristics and our outcomes, engagement in preventive behaviors. In these unadjusted models, older age (i.e., 50-59 years and 60+, vs. 18-29 years) emerged as protective for being in the low engagement versus the mixed engagement class (ORs 0.51 [95\% CI: $0.33-0.80$ ] and 0.23 [95\% CI: 0.15-0.35], respectively). Those aged 60 and older were also less likely 
Table 1 Demographic characteristics of UAS sample at baseline (March 10 through March 31)

\begin{tabular}{|c|c|c|}
\hline $\begin{array}{l}\text { Variable } \\
\text { Sociodemographic characteristics }\end{array}$ & Level & $\mathrm{N}(\%)$ \\
\hline \multirow[t]{5}{*}{ Age category } & $18-29$ & $513(11.5 \%)$ \\
\hline & $30-39$ & $1048(23.6 \%)$ \\
\hline & $40-49$ & $688(15.5 \%)$ \\
\hline & $50-59$ & $794(17.9 \%)$ \\
\hline & $60+$ & $1402(31.5 \%)$ \\
\hline \multirow[t]{2}{*}{ Sex } & Female & $2227(50.1 \%)$ \\
\hline & Male & $2218(49.9 \%)$ \\
\hline \multirow[t]{4}{*}{ Race/ethnicity } & White, non-Hispanic & $2925(65.8 \%)$ \\
\hline & Black, non-Hispanic & $452(10.2 \%)$ \\
\hline & Hispanic/Latino & $630(14.2 \%)$ \\
\hline & Other, non-Hispanic & $434(9.8 \%)$ \\
\hline \multirow[t]{4}{*}{ Educational attainment } & High school or less & $1491(33.6 \%)$ \\
\hline & Some college/Associate's degree & $1250(28.1 \%)$ \\
\hline & Bachelor's degree & $921(20.7 \%)$ \\
\hline & Graduate degree & $782(17.6 \%)$ \\
\hline \multirow[t]{5}{*}{ Geographic region } & Northeast & $796(17.9 \%)$ \\
\hline & Midwest & $903(20.3 \%)$ \\
\hline & South & $1671(37.6 \%)$ \\
\hline & West & $1075(24.2 \%)$ \\
\hline & Health beliefs & \\
\hline \multirow[t]{3}{*}{ Perceived risk of infection } & No risk $(0 \%)$ & $786(17.7 \%)$ \\
\hline & Low risk $(0.1-20 \%)$ & $1904(42.8 \%)$ \\
\hline & High risk $(20.1-100 \%)$ & $1754(39.5 \%)$ \\
\hline \multirow[t]{3}{*}{ Perceived risk of death if infected } & No risk $(0 \%)$ & $921(20.7 \%)$ \\
\hline & Low risk $(0.1-20 \%)$ & $2471(55.6 \%)$ \\
\hline & High risk $(20.1-100 \%)$ & $1053(23.7 \%)$ \\
\hline \multicolumn{2}{|c|}{ Perceived effectiveness of recommended behaviors (range 3-12) } & $M=9.68(\mathrm{SD}=0.036)$ \\
\hline
\end{tabular}

to be in the high engagement class relative to the mixed engagement class (OR: 0.56 [95\% CI: 0.35-0.91]). Males had significantly lower odds of being in the high engagement class (OR: 0.68, [95\% CI: 0.54-0.86]) than the mixed engagement class. When compared to White individuals, all other racial/ethnic groups (Black, non-Hispanic; Hispanic/Latino; and Other, non-Hispanic) showed significantly higher odds of being in the high engagement group (ORs between 2.54 and 3.18 , all statistically significant) relative to the mixed engagement group. Those with a higher level of educational attainment had reduced odds of being in the low engagement group (OR: 0.49 [95\% CI: 0.35-0.69] for bachelor's degree, 0.35 [95\% CI: 0.23-0.54] for graduate degree) and higher odds of being in the high engagement group (OR: 1.49 [95\% CI: 1.06-2.08]) relative to the mixed engagement class. When compared to individuals in the Northeast, those in the Midwest and South showed significantly higher odds of being in the low engagement group (ORs 1.86 [95\% CI:

Table 2 Summary of model fit criteria

\begin{tabular}{|c|c|c|c|c|c|c|c|}
\hline $\begin{array}{l}\text { Number } \\
\text { of classes }\end{array}$ & Smallest class size & Log likelihood & $\begin{array}{l}\text { Akaike information } \\
\text { criteria (AIC) }\end{array}$ & $\begin{array}{l}\text { Bayesian information } \\
\text { criteria (BIC) }\end{array}$ & $\begin{array}{l}\text { Sample-size adjusted } \\
\text { BIC }\end{array}$ & Entropy & $\begin{array}{l}\text { Lo-Mendell-Rubin likelihood } \\
\text { ratio test (LRT) }\end{array}$ \\
\hline 2 & 0.28437 & $21,395.022$ & $42,740.284$ & $42,874.674$ & $42,807.944$ & 0.781 & 4182.607 \\
\hline 3 & 0.19758 & $20,619.563$ & $41,303.126$ & $41,507.912$ & $41,406.228$ & 0.791 & 1443.534 \\
\hline 4 & 0.03582 & $20,507.73$ & $41,101.46$ & $41,376.64$ & $41,240.003$ & 0.791 & 221.272 \\
\hline 5 & 0.03540 & $20,456.886$ & $41,021.772$ & $41,367.347$ & $41,195.756$ & 0.794 & 100.599 \\
\hline 6 & 0.03724 & $20,418.031$ & $40,966.062$ & $41,382.031$ & $41,175.487$ & 0.695 & 76.878 \\
\hline
\end{tabular}




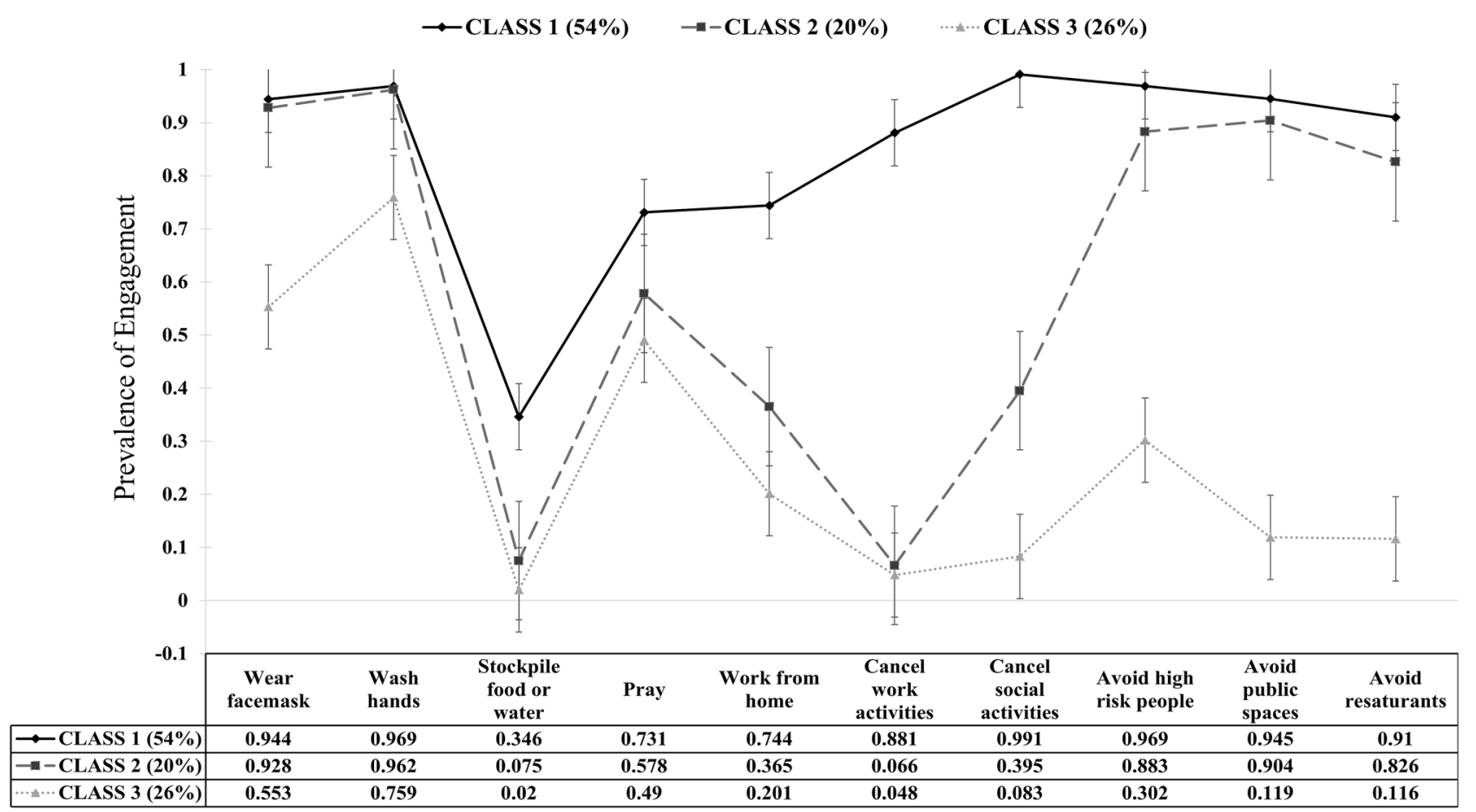

Fig. 1 Probability of engaging in preventive behaviors by latent class

1.24-2.78]) and 1.94 [95\% CI: 1.31-2.89], respectively) relative to the mixed engagement group. Similarly, when compared to individuals in the Northeast, individuals in the
South had a higher odds of being in the high engagement group (OR: 1.80 [95\% CI: $1.25-2.58]$ ), relative to the mixed engagement group.

Table 3 Multinomial logistic regression of bivariate associations between sociodemographic characteristics and latent class membership

\begin{tabular}{|c|c|c|c|c|c|}
\hline & \multicolumn{2}{|c|}{$\begin{array}{l}\text { Low engagement in preventive } \\
\text { behaviors }\end{array}$} & \multirow{2}{*}{$\begin{array}{l}\text { Mixed engagement in } \\
\text { preventive behaviors }\end{array}$} & \multicolumn{2}{|c|}{$\begin{array}{l}\text { High engagement in preventive } \\
\text { behaviors }\end{array}$} \\
\hline & OR $[9$ & & & OR $[95$ & \\
\hline \multicolumn{6}{|l|}{ Age category (ref: 18-29) } \\
\hline $30-39$ & 0.66 & $(0.43,1.03)$ & -- & 0.94 & $(0.59,1.49)$ \\
\hline $40-49$ & 0.74 & $(0.47,1.15)$ & -- & 0.96 & $(0.60,1.56)$ \\
\hline $50-59$ & 0.51 & $(0.33,0.80)^{*}$ & -- & 0.78 & $(0.49,1.25)$ \\
\hline $60+$ & 0.23 & $(0.15,0.35)^{*}$ & -- & 0.43 & $(0.28,0.67)^{*}$ \\
\hline \multicolumn{6}{|l|}{ Sex (ref: female) } \\
\hline Male & 1.21 & $(0.96,1.53)$ & -- & 0.68 & $(0.54,0.86)^{*}$ \\
\hline \multicolumn{6}{|c|}{ Race/ethnicity (ref: white, non-Hispanic) } \\
\hline Black, non-Hispanic & 0.62 & $(0.36,1.07)$ & -- & 3.11 & $(2.14,4.53)^{*}$ \\
\hline Hispanic/Latino & 1.16 & $(0.75,1.80)$ & -- & 3.18 & $(2.20,4.61)^{*}$ \\
\hline Other & 0.76 & $(0.46,1.27)$ & -- & 2.54 & $(1.70,3.79)^{*}$ \\
\hline \multicolumn{6}{|c|}{ Educational attainment (ref: high school or less) } \\
\hline Some college/Associate's & 0.88 & $(0.66,1.17)$ & -- & 1.04 & $(0.76,1.42)$ \\
\hline Bachelor's degree & 0.49 & $(0.35,0.69)^{*}$ & -- & 0.76 & $(0.54,1.06)$ \\
\hline Graduate degree & 0.35 & $(0.23,0.54)^{*}$ & -- & 1.49 & $(1.06,2.08)^{*}$ \\
\hline \multicolumn{6}{|c|}{ Geographic region (ref: Northeast) } \\
\hline Midwest & 1.86 & $(1.24,2.78)$ & -- & 0.93 & $(0.62,1.39)$ \\
\hline South & 1.94 & $(1.31,2.89)$ & -- & 1.80 & $(1.25,2.58)$ \\
\hline West & 1.31 & $(0.86,1.98)$ & -- & 1.42 & $(0.99,2.04)$ \\
\hline
\end{tabular}

*Indicates statistical significance at a $p<0.05$ 


\section{Health Beliefs and Classes of Preventive Behaviors}

Respondents with "low" or "high" perceived risk of infection were significantly less likely than those who perceived no risk to be in the low vs. mixed engagement class; odds ratios were 0.51 for low risk (95\% CI: $0.37-0.71)$ and 0.58 for high risk (95\% CI: 0.41-0.81) (Table 4, unadjusted models). Similarly, those in the "low risk" group compared to the "no risk" group were 0.57 times less likely to be in the high engagement class, relative to the mixed engagement class (95\% CI: 0.41-0.80). The other health beliefs were also associated with lower odds of class membership, but only for the low engagement class relative to the mixed engagement class. Specifically, individuals who perceived a low risk of death from infection were 0.61 times less likely to be in the low engagement class, relative to the mixed engagement class (95\% CI: $0.45-0.81$ ), whereas those with high perceived risk of death were 0.54 times less likely to be in the low vs. mixed engagement class (95\% CI: $0.37,0.77$. Similarly, for every one-point increase in perceived effectiveness of recommended preventive behaviors, the likelihood of being in the low engagement group decreased by $11 \%$ (OR: 0.89, 95\% CI: 0.83-0.95).

In the final series of models, we assessed the relationship between health beliefs and preventive behaviors after adjusting for sociodemographic characteristics. Results were largely similar to the unadjusted models (Table 4, adjusted models). Notable differences among sociodemographic characteristics include the following: (1) after adjustment, males, relative to females, had a statistically significant higher odds of being in the low engagement class, compared to the mixed engagement class in all health beliefs models (ORs: 1.49-1.54), and (2) Black, non-Hispanic individuals, relative to white, non-Hispanic individuals, had significantly lower odds of being in the low engagement class, relative to the mixed engagement class, in all health belief models (ORs: 0.39-0.42). No differences in significance were detected among health beliefs. A summary of these results is available in Tables 3 and 4.

\section{Discussion}

The objectives of this study were to (1) characterize patterns of preventive behaviors three months after the COVID-19 pandemic was declared a national emergency in the USA and (2) identify how health beliefs and sociodemographic characteristics are associated with preventive behaviors. Using data collected 3 months after the pandemic was declared a national emergency, three latent classes of preventive behavior emerged based on patterns of engagement, i.e., low engagement, high engagement, and mixed engagement. Respondents in the low and high engagement classes reported the lowest and highest numbers of behaviors,

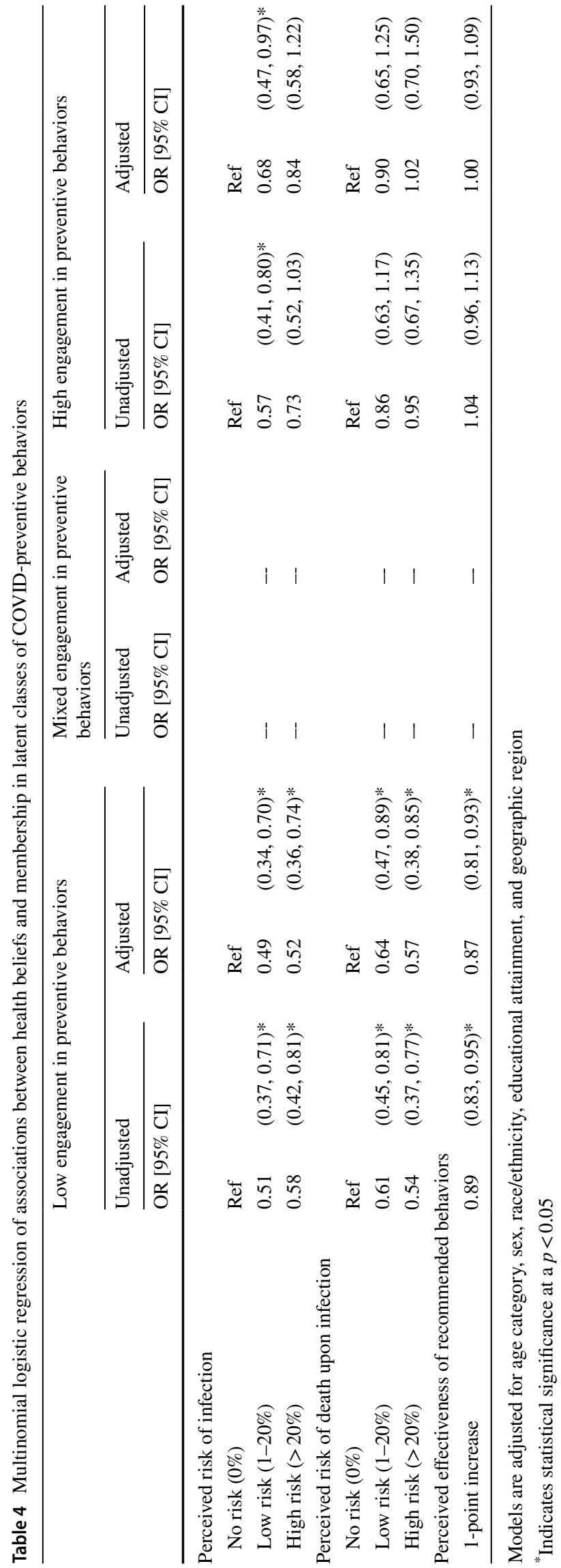


respectively. Those in the mixed-engagement class endorsed most preventive behaviors but were less likely to endorse preventive behaviors related to social distancing (e.g., working from home, canceling social activities or trips). These findings highlight the heterogeneous nature of engagement in preventive behaviors during the pandemic.

\section{Sociodemographic Characteristics and COVID-Preventive Behaviors}

The findings revealed differences in class membership linked to age category, sex, racial/ethnic group, education level, and census region, corroborating recent research that highlights group differences in adoption of recommended behaviors (Alsan et al., 2020; Wise et al., 2020). Since the beginning of the outbreak, older adults have been highlighted as a susceptible population. Our work shows that-compared to 18-29-year-olds-older adults have a lower odds of being in the low and high engagement classes relative to the mixed engagement class. Older adults' lower odds of being in the low engagement class may be a consequence of their strong endorsement of preventive behaviors (Hutchins et al., 2020). Older adults report mental distress (Koma et al., 2020) surrounding the pandemic that likely encourages their adherence to strict guidelines. Yet, in our study, older adults also had a higher odds of being situated in the mixed engagement class relative to the high engagement class, which could be due to the conditional nature of the items that the mixed engagement class fail to endorse (i.e., working from home and cancelling activities). Older adults are more likely to be retired than younger adults and would not need to work from home. Similarly, they are less likely to have work or social activities that would need to be cancelled or rescheduled (Marcum, 2012), suggesting that they are also unlikely to endorse these items. Therefore, it is not surprising that we see older adults primarily situated in the mixed engagement group.

When compared to White individuals, all other racial/ ethnic groups (Black, non-Hispanic; Hispanic/Latino; and Other, non-Hispanic) show significantly higher odds of reporting engagement in all preventive behaviors, including working from home and cancelling social and workrelated activities. This finding is particularly notable given the racial inequities that minority communities face, such as racial stereotyping (Christiani et al., 2020), which has been linked with mask wearing, and disproportionate involvement in service occupations, which are limited in opportunities for tele-working. Racial differences in support for and endorsement of preventive behaviors have been highlighted throughout the pandemic (Gecewicz, 2020; Igielnik, 2020). These racial and ethnic differences may be linked to the heightened inequities marginalized racial groups experience such as two to three times the number of hospitalizations and deaths following COVID-19 infection (CDC, 2020). In particular, it has been hypothesized that Black Americans are at greater risk for COVID-19 exposure due to their representation in service occupations and a high likelihood of living in densely populated areas (Egede \& Walker, 2020). These risk factors may be associated with a higher likelihood of engaging in prevention efforts per increased perceptions of susceptibility.

Other sociodemographic characteristics, including education level, sex, and census region are also significantly associated with class membership. In general, individuals with higher levels of education (e.g., graduate degree) are significantly more likely to engage in preventive behaviors, as are females. Differences in mask wearing among various education levels (Kramer, 2020) and sexes have already been documented (Brenan, 2020). Distinctions in COVID-19 restrictions are common across states. States in the Northeast region consistently demonstrate higher levels of mask-wearing (Katz et al., 2020) and COVID-related policies, with 8 of 9 Northeastern states among the top 20 states with most COVID19 restrictions (McCann, 2021). Almost all Southern and Midwestern states show lower rates of COVID-19 prevention behaviors and policies, which aligns with our finding that they are more likely to be in the low engagement group. Engagement and restrictions among Western states are mixed, with the Pacific West (i.e., California, Oregon, and Washington) among the most stringent, and other Western states among the least. Therefore, it is not surprising that we see no significant differences in prevention behavior patterns between the Northeast and West. However, our findings also show that individuals in Southern states, relative to Northeastern states, are more likely to be in the high engagement group relative to the mixed engagement group. This is likely due to confounding by demographic variables, such as differences in age and race breakdown by region, as the finding is null in adjusted models. Taken together, these findings highlight novel patterns of behavior adoption among sociodemographic subgroups, particularly in terms of those most likely to be situated in the mixed engagement class and demonstrate that some behaviors may be adopted based on convenience or ability rather than strictly following health beliefs. Importantly, these sociodemographic factors may moderate associations between health beliefs and behavior. Future studies should consider running stratified analyses to determine whether these associations differ across demographic subgroups.

\section{Health Beliefs and COVID-Preventive Behaviors}

Our study showcases differences in class membership linked to health beliefs in March, including perceived risk of infection, perceived risk of death following infection, and perceived effectiveness of recommended preventive behaviors. These 
findings indicate the importance of targeting health beliefs as a step toward promoting COVID-preventive behaviors such as mask wearing and handwashing (Van den Broucke, 2020). Associations between health beliefs and class membership follow the framework of the HBM. In alignment with the model, individuals who believed they were more susceptible to infection or death were less likely to be in the low engagement class, relative to the mixed engagement class. Similarly, those who believed that the $\mathrm{CDC}$ recommendations were an effective prevention strategy for COVID-19 were more likely to be in a latent class that endorsed more preventive behaviors. However, somewhat surprisingly, individuals with a "low" perceived risk of infection as compared to "no risk" were less likely to be in the high engagement class than in the mixed engagement class. This may be due to a cyclic association: individuals in this category may perceive that they have a low risk of infection (rather than "no risk") because they cannot endorse certain behaviors (e.g., they have to go to work or have to perform work activities to keep a job). Following this logic, these individuals would be more likely to be in the mixed class because they are not endorsing those preventive behaviors. Together, the associations between health beliefs and the latent class analysis follow the model assumptions: individuals who believe they are susceptible to infection or death, or who believe in the effectiveness of preventive behaviors, show increased likelihood to take action to prevent the spread of COVID-19.

\section{Strengths and Limitations}

Our study has many strengths, including use of a large and nationally representative sample, assessment of a broad set of preventive behaviors and health beliefs, and multiple waves. However, this study also has several limitations worth noting. First, there are differences in mandates and norms about wearing masks across geographic settings. Additionally, some areas have opportunities designed to facilitate high-risk people, such as special grocery store hours for vulnerable populations. For example, special grocery store hours may allow older adults to feel less at-risk and engage in more prevention behaviors (e.g., social distancing) than their peers who do not have these accommodations. We were unable to account for these differences in our analysis but recognize that they may influence engagement in preventive behaviors and perceived susceptibility and severity. Second, some prevention behaviors were not applicable to all participants, such as cancelling work or social activities, which may have led to misclassification. For example, if a person reported not working from home because they do not have a job, they may be relegated to a lower engagement class. While our models adjusted for potential misclassification, it is important to highlight that some findings, such as those in adults aged 60 and older, may be influenced by the conditional nature of these items. Third, we did not adjust for news source and consumption, which have been associated with COVID-related health beliefs (Dhanani \& Franz, 2020). We acknowledge that additional work will be necessary to understand how news consumption influences prevention behaviors. Finally, the dataset did not include items to assess barriers to engaging in the preventive behaviors. Although many of the preventive behaviors were not complex or resourceintensive, it is likely that some of the people who did not report specific behaviors experienced barriers to engagement, such as work requirements. Investigating these potential mediators is an important direction for future studies.

\section{Conclusion}

These data are unique in that they capture the preventive behaviors of the US population approximately 3 months into the US response to the pandemic (May/June), when most federal and state guidelines had been lifted and people were no longer required to engage in preventive behaviors. The Health Belief Model proved to be a useful framework to identify health beliefs that were associated with class membership; this study supports previous research showing differences in endorsement of preventive behaviors (particularly mask wearing), and highlights different patterns of prevention behaviors among distinct sociodemographic groups, who may experience systemic inequalities (e.g., disproportionate participation in service occupations) that limit ability to engage in prevention behaviors, in addition to different patterns of engagement among individuals with different health beliefs. These findings highlight the potential utility of using health beliefs to inform health communication campaigns and other prevention efforts, and also suggest that HBM can be a useful framework for understanding COVID-related health behaviors. Our findings can be used by practitioners to identify systemic barriers to engagement or develop health communication campaigns targeted to a specific demographic subgroup (e.g., a social media campaign for emerging adults) to reduce the spread of COVID-19 and future pandemics.

Funding The Understanding America Study is funded from several sources, including the Social Security Administration and the National Institute on Aging under grant 5U01AG054580. The survey that collected the mental health and COVID-19-related data used in this paper was funded by the Center for Economic and Social Research at USC. Work on the current manuscript was in part supported by a RAPID grant from the National Science Foundation (grant number 2028683). Ms. Smail is supported by the NIA Epidemiology and Biostatistics of Aging Training Program (T32AG000247). Dr. Schneider is supported by NIDA grant 5T32DA007292-25, and Dr. DeLong is supported by NIH/NIAID grant T32AI102623. Dr. Sanders is also supported by the National Institutes of Health (NIDA grant R01DA043089), and the Johns Hopkins University Center for AIDS Research (P30AI094189) supports the Adolescent and Young Adult Scientific Working Group, whose membership contributed to conceptualization and manuscript preparation. 


\section{Declarations}

Consent to Participate Households who log into the UAS website are asked to agree to an online consent before they take the first survey.

Conflict of Interest All the authors have no potential conflicts of interest to disclose. The project described in this paper relies on data from survey(s) administered by the Understanding America Study, which is maintained by the Center for Economic and Social Research (CESR) at the University of Southern California. The content of this paper is solely the responsibility of the authors and does not necessarily represent the official views of USC or UAS. For any questions or more information about the UAS, contact Tania Gutsche, Project and Panel Manager, Center for Economic and Social Research, University of Southern California, at tgutsche@usc.edu.

\section{References}

Alsan, M., Stantcheva, S., Yang, D., \& Cutler, D. (2020). Disparities in coronavirus 2019 reported incidence, knowledge, and behavior among US adults. JAMA Network Open, 3, e2012403. https://doi. org/10.1001/jamanetworkopen.2020.12403

Asparouhov, T., \& Muthén, B. (2014). Auxiliary variables in mixture modeling: Three-step approaches using mplus. Structural Equation Modeling, 21(3), 329-341. https://doi.org/10.1080/10705511. 2014.915181

Brenan, M. (2020). Americans' face mask usage varies greatly by demographics. Retrieved from https://news.gallup.com/poll/315590/ americans-face-mask-usage-varies-greatly-demographics.aspx

CDC. (2020). Coronavirus disease 2019 (COVID-19) - Prevention \& treatment . Retrieved from https://www.cdc.gov/coronavirus/ 2019-ncov/prevent-getting-sick/prevention.html

Christiani, L., Clark, C. J., Greene, S., Hetherington, M. J., \& Wager, E. (2020). Masks and racial stereotypes in a pandemic: The case for surgical masks. Rochester, NY. https://doi.org/10.2139/ssrn.3636540 Retrieved from https://papers.ssrn.com/abstract $=3636540$

Dhanani, L. Y., \& Franz, B. (2020). The role of news consumption and trust in public health leadership in shaping COVID-19 knowledge and prejudice. Frontiers in Psychology, 11, 560828.

Egede, L. E., \& Walker, R. J. (2020). Structural racism, social risk factors, and covid-19 - A dangerous convergence for black Americans. The New England Journal of Medicine, 383, e77. https://doi. org/10.1056/NEJMp2023616

Gecewicz, C. (2020). Amid pandemic, black and Hispanic worshippers more concerned about safety of in-person religious services. Retrieved from https://www.pewresearch.org/fact-tank/2020/08/07/ amid-pandemic-black-and-hispanic-worshippers-more-concernedabout-safety-of-in-person-religious-services/

Glanz, K., Rimer, B. K., \& Viswanath, K. (Eds.). (2015). Health behavior: Theory, research, and practice (5th ed.) Jossey-Bass/Wiley.

Goldberg, M. H., Gustafson, A., Maibach, E. W., Ballew, M. T., Bergquist, P., Kotcher, J. E., \& Leiserowitz, A. (2020). Maskwearing increased after a government recommendation: A natural experiment in the U.S. during the COVID-19 pandemic. Frontiers in Communication, 5. https://doi.org/10.3389/fcomm.2020.00044

Grossman, G., Kim, S., Rexer, J., \& Thirumurthy, H. (2020). Political partisanship influences behavioral responses to governors' recommendations for COVID-19 prevention in the United States. Rochester, NY. https://doi.org/10.2139/ssrn.3578695 Retrieved from https://papers.ssrn.com/abstract $=3578695$

Hutchins, H. J., Wolff, B., Leeb, R., Ko, J., Odom, E., Willey, J., \& Bitsko, R. (2020). COVID-19 mitigation behaviors by age group United States, April-June 2020. MMWR. Morbidity and Mortality
Weekly Report, 69, 1584-1590. https://doi.org/10.15585/mmwr. mm6943e4

Igielnik, R. (2020, June 23). Most Americans say they regularly wore a mask in stores in the past month; fewer see others doing it. Retrieved from https://www.pewresearch.org/fact-tank/2020/06/ 23/most-americans-say-they-regularly-wore-a-mask-in-stores-inthe-past-month-fewer-see-others-doing-it/

Johns Hopkins Coronavirus Resource Center. (2020). COVID-19 United States cases by state and county Johns Hopkins University \& Medicine.

Katz, J., Sanger-Katz, M., \& Quealy, K. (2020). A detailed map of who is wearing masks in the U.S. The Upshot - New York Times. Retrieved from https://www.nytimes.com/interactive/2020/07/17/ upshot/coronavirus-face-mask-map.html

Koma, W., True, S., Fuglesten Biniek, J., Cubanski, J., Orgera, K., \& Garfield, R. (2020). One in four older adults report anxiety or depression amid the COVID-19 pandemic. Retrieved from https://www. kff.org/medicare/issue-brief/one-in-four-older-adults-report-anxietyor-depression-amid-the-covid-19-pandemic/

Kramer, S. (2020, August 27). More Americans say they are regularly wearing masks in stores and other businesses. Retrieved from https://www.pewresearch.org/fact-tank/2020/08/27/moreamericans-say-they-are-regularly-wearing-masks-in-stores-andother-businesses/

Lavrakas, P. J. (2008). Encyclopedia of survey research methods (vols. 1-0). https://doi.org/10.4135/9781412963947

Marcum, C. S. (2012). Age differences in daily social activities. Research on Aging, 35, 612-640. https://doi.org/10.1177/ 0164027512453468

McCann, A. (2021). States with the fewest coronavirus restrictions. https://wallethub.com/edu/states-coronavirus-restrictions/73818

Muthen, L. K., \& Muthen, B. O. (2017). Mplus user's guide. Eighth edition.[computer software]. Los Engeles, CA: Muthen \& Muthen.

Nylund-Gibson, K., \& Choi, A. Y. (2018). Ten frequently asked questions about latent class analysis. Translational Issues in Psychological Science, 4, 440-461.

Raifman, J., Nocka, K., Jones, D., Bor, J., Lipson, S., Jay, J., \& Chan, P. (2020). COVID-19 US state policy database. Retrieved from www.tinyurl.com/statepolicies

Rosenstock, I. M. (1974). The health belief model and preventive health behavior. Health Education Monographs, 2, 354-386. https://doi.org/10.1177/109019817400200405

Statacorp. (2017). Stata statistical software: Release 15. [computer software]. College Station, TX: StataCorp LLC.

The University of Southern California. (2020). Understanding America study. Retrieved from https://uasdata.usc.edu/index.php

US Bureau of Labor Statistics. (2021). Current population survey (CPS). Retrieved from https://www.bls.gov/cps/

US Census Bureau. (2013). Census regions and divisions of the United States. https://www2.census.gov/geo/pdfs/maps-data/ maps/reference/us_regdiv.pdf

Van den Broucke, S. (2020). Why health promotion matters to the COVID-19 pandemic, and vice versa. Health Promotion International, 35, 181-186. https://doi.org/10.1093/heapro/daaa042.

Wise, T., Zbozinek, T. D., Michelini, G., Hagan, C. C., \& Mobbs, D. (2020). Changes in risk perception and protective behavior during the first week of the COVID-19 pandemic in the united states. https://doi.org/10.31234/osf.io/dz428

Zine, J. (2020). Unmasking the racial politics of the coronavirus pandemic. Retrieved from http://theconversation.com/unmasking-theracial-politics-of-the-coronavirus-pandemic-139011

Publisher's Note Springer Nature remains neutral with regard to jurisdictional claims in published maps and institutional affiliations. 